Focus. I turn off most mobile notifications and set my phone to "do not disturb." I stay off social media when working, and (worth emphasizing again) I don't check email as a distraction (new work will manifest, and if I can't dedicate 10-20 minutes to process, then this lingers and distracts). Any emails related to a joint project are printed to pdf and put in a folder related to that project as they arrive so that I don't need to check email when working.

Automate. I coordinate our weekly Algebra and Number Theory research seminar. For each outside speaker, I send 5-6 emails (announcing the seminar, requesting that the staff make and post flyers, processing reimbursement, etc.). The emails are basically the same every week, with a few variables; I have a bash script that writes the emails for me.

Saying no. Initially I accepted every reasonable referee request. Eventually, several senior colleagues explained that they turn down many requests. Good citizens seem to referee roughly three papers for each paper they submit, and I try to stick to that (even if the paper looks interesting and I'm an appropriate referee).

It's difficult to say no, and psychologically, I needed "permission" to start declining requests. It was helpful that my department's service expectations were clear and direct. Similarly, I found writing for Mathematical Reviews stressful and difficult but organizing conferences enjoyable and natural; these days I do more of the latter.

Efficiency. I could fill another article with habits and routines that create and protect time.

I bike to work: it takes about 25 minutes, compared to 35-40 to drive and park. Our department has a shower on my floor. Cycling doubles as cardio and boosts my mood and mental health.

Each semester I poll students to find a time that accommodates everyone who might possibly attend office hours. I reserve a classroom for office hours. Students can show up even if they don't have focused questions, and I can leave immediately when finished. (In any case I can't fit more than 3-4 people in my office.)

I scan my lecture notes after each class; I include the date, lecture number, and course number in the filename, and keep a terse outline of what I covered each lecture. Teaching a course a second (or ninth...) time is a breeze, and this type of organization creates time and space to focus on improving the course. And if I'm stuck somewhere (e.g., delayed flight), I can access the notes via Dropbox and use that time for review and preparation.

In fact, I scan everything; my office is across from the printer/scanner, and Dropbox has a useful mobile app (if I have a meal receipt that needs to be reimbursed, I scan it immediately). If I give a chalkboard talk, I scan the notes (and put the scan somewhere easy to find again).

Discuss! I've had countless conversations about these topics (at tea, conference dinners, in hallways) and have benefited greatly. Our profession tends to pile on extra work with little guidance, and reaching out to experienced colleagues can be productive and therapeutic.

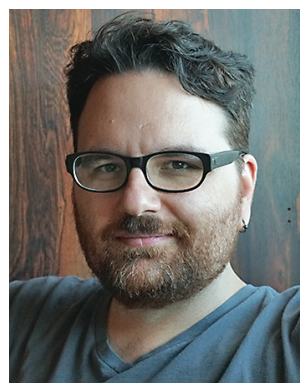

David Zureick-Brown

Credits

Author photo is courtesy of Sarah Zureick-Brown.

\section{How to Read a Research Paper}

\section{Matt Baker}

Before attempting to read a research paper, I recommend first deciding why you want to read it, what you hope to get out of the paper, and how much time you're willing to commit. Then place the paper into one of the following three categories:

- Speed Read: A paper whose introduction you plan to read in order to get an overview of the results and then possibly skim further.

- Substantial Skim: A paper that you plan to skim all the way through, perhaps reading certain parts in detail.

- Deep Dive: A paper that you wish to thoroughly study and understand.

It's useful to have different categories because there's so much interesting math research produced every day, and it's impossible to keep up with everything. I go through the arXiv preprint listings in three different categories almost religiously every single morning. I get email notifications for all new postings and revisions in these categories, and I make an effort most days to "speed read" at least one new paper while drinking my morning coffee. I also bookmark papers to come back to later (although to be honest, I end up not having time to come back to many of these). Skimming through the arXiv abstracts in the daily digest and then speed reading at least one paper a day keeps me feeling in touch with what's happening in the fields I'm most interested in.

Matt Baker is a professor of mathematics at Georgia Institute of Technology. His email address is mbaker@math.gatech. edu.

DOI: https://dx.doi.org/10.1090/noti2077 
With a Speed Read, I don't spend a lot of time trying to come to terms with complex definitions or understand precisely why the hypotheses in various theorems are what they are; I just want to get an overall impression of the paper and learn something new, even if it's mostly superficial knowledge. This kind of reading will take me about 15 minutes for an average paper.

If the paper seems really interesting and I'm wanting to read and learn more, I'll upgrade it to the "Substantial Skim" category and come back to it later in more detail. I've found that over time, the collection of quick impressions I obtain through my Speed Reads actually provides a reasonably deep foundation for understanding various bits of the mathematical landscape at large.

For a smaller number of papers (roughly one a week, in my case, though the variance is high), I will do a more Substantial Skim. This involves reading through all of the definitions and statements, and at least some of the proofs, in the whole paper and making an attempt to actually understand what's going on. My goal here is to embed the paper firmly enough into my mind that I'll be able to incorporate some of the ideas into my thinking later on. When I read a paper in this way, I will sometimes jot down key definitions or statements (either in a physical notebook or Evernote file), and I might go through certain arguments carefully in order to understand particular points in detail. However, I don't make an attempt to check the paper for correctness or try to fully understand all of the technical points. This is actually my favorite category of reading, because I learn a tremendous amount without having to put in too many hours of work. Depending on the complexity of the paper, this kind of reading might take me anywhere between one and three hours per 10 pages.

Finally, there's the Deep Dive. These days, I probably do this for only about one paper every month or two (though when I was younger and less overwhelmed with responsibilities, it was quite a bit more frequent). Here, I spend as much time as it takes to understand the definitions, the conditions of the theorems, and the logic behind the arguments. I will sometimes write out detailed notes in a notebook, and as often as possible I'll stop reading and see if I can figure out the next step myself. If I'm really interested in understanding the paper and retaining the knowledge, I'll try to explain the results to someone else; there's no better way than teaching to internalize complex information! I'll also look up background facts in the references and, as long as it doesn't lead to an exponential recursion, try to understand that material as well. And I'll keep a list of typos or other errors I find and send it to the author as appropriate. This kind of reading can take anywhere from 15 minutes to a couple of hours per page, depending on the subject matter and how familiar I am with the underlying concepts.

To be perfectly honest, these days I find the time for a Deep Dive only when a paper is either directly relevant to my current research or I've agreed to referee it. But that's an artifact of choices I've made and priorities I've set in my own life, and of course if you really want to build up a formidable knowledge base, you should take Deep Dives as often as possible. If, like me, you're constantly feeling busy and overwhelmed, another way to "read" a paper is to assign it to a student to explain to you! Or put together a study group and parcel out the task to various students and colleagues. I did this recently with the paper "Lorentzian Polynomials" by Petter Brändén and June Huh over the course of a whole semester. (For an example of how to organize such a seminar, see https://sites.google .com/view/gtlorentzian.)

One other thing I think is important is to read widely and try to push the boundaries of your understanding. Especially with Speed Reads and Substantial Skims, don't just read a bunch of papers about the same topic all the time. By reading about different topics within a short time frame, your brain will automatically start to explore connections and begin thinking "outside the box."

This is my own personal approach to reading math papers, and I don't claim that the same techniques will work for everyone. But I do think that every working mathematician needs to develop a system for reading research papers in order to attain, over time, both the breadth and depth of knowledge required to keep up with the relentless but thrilling march of progress in modern mathematics.

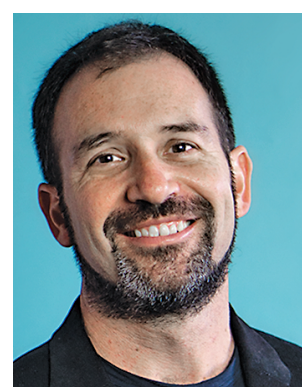

Matt Baker

Credits

Author photo is courtesy of the author. 